\title{
La evaluación en la Educación Patrimonial. 20 años de investigaciones y Congresos de ICOM
}

\author{
Roser Calaf, Sué Gutiérrez Berciano y Miguel Ángel Suárez Suárez \\ Universidad de Oviedo - España
}

\section{RESUMEN}

Los esfuerzos por clasificar son mecanismos lógicos empleados para la formalización de esquemas o modelos que permitan comprender la realidad. El carácter de clasificación impera en ambos campos. Con la intención de reivindicar la importancia de la filogénesis, y recuperando esta idea de ordenamiento, se realiza un estudio bibliográfico de la producción científica de la Educación Patrimonial. Se realiza una revisión documental y análisis de contenido de Tesis Doctorales y Congresos específicos organizados por ICOM en las últimas dos décadas. Desde una aproximación descriptiva, se identifica el perfil de las evaluaciones desarrolladas (finalidad, muestra de estudio, metodología y técnicas empleadas) cuestiones que concretan la visión panorámica de la evaluación en este campo de conocimiento. Los resultados muestran cómo la evaluación ha tenido un papel secundario en el ámbito de la educación patrimonial hasta épocas recientes, cuando parece que la preocupación por la calidad ha influido en el interés creciente por la evaluación.

Palabras clave: Educación Patrimonial, Evaluación, Revisión bibliográfica, Análisis documental, Tesis Doctoral.

\section{Evaluation in the Heritage Education. 20 years of research and congresses of ICOM}

\section{ABSTRACT}

The efforts to classify are logical mechanisms to understand reality. In Natural Sciences, a taxon specifies its phylogeny (Kingdom), class, order, family, gender and species. In this way, it is intended to discover patterns for formalization. In Social Sciences, saving the distances, the tendency is similar and it is oriented to discover the sociocultural, political, economic codes... for the formalization of schemes or models. The classification character prevails in both fields. With the intention of claiming the importance of phylogenesis, and recovering this idea of ordering, a bibliographical study of the scientific production of Heritage Education is carried out. A documentary review and analysis of the content of specific Doctoral Theses and Congresses organized by ICOM in the last two decades is carried out. From a descriptive approach, the profile of the evaluations developed (purpose, study sample, methodology and techniques used) issues that specify the panoramic vision of the evaluation in this field of knowledge is identified. The results show how the evaluation has had a secondary role in the field of heritage education until recent times, when it seems that the concern for quality has influenced the growing interest in the evaluation.

Keywords: Heritage Education, Evaluation, Bibliographic review, Documentary analysis, Doctoral Thesis. 


\section{Introducción y antecedentes}

El vínculo planteado entre evaluación y Educación Patrimonial (EP) requiere de una serie de especificaciones previas. Ambos ámbitos tienen carácter interdisciplinar que exigen una justificación del planteamiento abordado en este estudio. Se plantea la Educación Patrimonial considerando los "programas educativos" desarrollados por los museos o sitios de patrimonio. Y por su parte, se entiende la evaluación desde una perspectiva educativa en la que convergen diversos modelos y enfoques que darán una respuesta coherente a la educación (Mark, Henry \& Julnes, 2000) en este caso en, para y por el patrimonio (Suárez, 2015; 2017). En definitiva, se persigue evidenciar la tendencia evaluativa que se ha venido ensayando en la acción educativa desarrollada en museos y sitios de patrimonio desde una perspectiva holística, acorde al concepto actual del término "programa" y "evaluación".

La evaluación no es un ámbito novedoso en el contexto museístico, tiene su arraigo en las áreas de Museografía y Museología sobre dispositivos museográficos. Bien, enfocada a conocer el "público diana" de esas exhibiciones, Powel (1930), al incentivo investigador del impacto de la exposición en el visitante de Abbey y Cameron, en década posterior. Para pasar a otra fase donde la evaluación toma impulso renovado y metodológico con Scriven (1967), que en la década posterior con los trabajos de Shettel y Screven optimizan para ofrecer un corpus técnico-sistemático, donde la evaluación se institucionaliza con la creación de la AAM (1906), VSA (1990), y los Observatorios de Públicos (Francia, 1990 o Quebec,1998).

Las evaluaciones de Harris Shettel y Chanler Screven tienen carácter psicoeducativo, su interés es la transmisión del mensaje expositivo. Para ello aplican los procedimientos de investigación educativa a la evaluación de exposiciones y proponen un enfoque de la evaluación centrado en objetivos (Pérez Santos, 2000: 29). Tanto Shettel como Screven son los pioneros en analizar la vertiente más educativa del museo. Ambos "denuncian" los vagos objetivos existentes en la planificación, situación que ha de ser superada, si el museo es un medio educativo, cuya misión principal es transmitir conocimientos y permitir el aprendizaje.

Otro de los factores que va a contribuir en la dispersión temática de la evaluación en el museo, es la diversidad de disciplinas que analizan su actividad. Desde la museología encargada de conocer las relaciones del individuo y su medio cultural en estos espacios, a la museografía, sociología, psicología, pedagogía (Alarcón, 2007 y Bitgood, 2011). El objeto de estudio de la evaluación de público cambia en función de la disciplina, el contexto y la cronología. Una tarea complicada sería ofrecer una taxonomía fehaciente al respecto. Las clasificaciones más aceptadas en este campo son la generadas por McManus (1996) y Bitgood y Shettel (1996), que autores como Pérez Santos (2000), Asensio, Pol y Gomis (2001) o Romanello (2015) han matizado.

En nuestro país una de las primeras iniciativas de investigación fue el proyecto de I+D+i Evaluación Cualitativa de Programas Educativos en Museos Españoles (http: / / ecpeme.com/) desarrollado desde la Universidad de Oviedo En la actualidad existen iniciativas institucionales destacables en materia de investigación y evaluación en EP (Fontal e Ibáñez, 2015): el Plan Nacional de Educación y Patrimonio, y el Observatorio de Educación Patrimonial (OEPE (http://www.oepe.es/), incluso el Laboratorio Permanente de Públicos (LLP). Las primeras mantienen el binomio Educación-Patrimonio como aliciente de las propuestas de investigación, innovación y formación. Mientras que el LLP se encarga de la elaboración de estudios de público de los museos de titularidad estatal. Esta retrospectiva puede situar a priori la trayectoria de la evaluación en museos en nuestro país.
El OEPE, dado el nombre de la institución y el trabajo realizado se entiende su labor de "vigilancia estratégica", la creación de redes de colaboración y el uso compartido del conocimiento (Angulo, 2009). Los observatorios no dejan de ser estaciones de análisis del campo sobre el que "observan", pero también "actores" de colaboración e intercambio de la información datada. Desde 2010 se ha puesto en marcha el Plan Nacional de Educación Patrimonial en colaboración con el Instituto del Patrimonio Cultural de España, y las administraciones de las comunidades autonómicas. En 2012 sus esfuerzos se han concentrado también en la difusión, formación, en la realización de Congresos, jornadas y cursos. Su red de trabajo se ha ampliado desde 2014 con la creación de la Red Internacional de EP, así como su apoyo a la Agrupación de barrios por la defensa del patrimonio (ABADEP). Es en estos años cuando la evaluación ha tomado fuerza como centro de interés en el Observatorio para el análisis de programas desde la perspectiva educativa identitaria del patrimonio, mediante planteamientos sociológicos, artísticos, incluso tecnológicos con el uso de apps (Fontal e Ibáñez-Etxeberría, 2017).

\section{Diseño}

Nuestra investigación pretende contribuir a esclarecer las relaciones existentes entre EP y evaluación, respondiendo a los siguientes objetivos:

a) Conocer cuál es el tipo de evaluación asociada habitualmente a los programas y estudios de Educación Patrimonial.

b) Evidenciar cómo han evolucionado los modelos de evaluación en el ámbito de la Educación Patrimonial y cuáles son sus retos.

c) Efectuar una aproximación descriptiva a los principales enfoques evaluativos desarrollados en la producción científica (Tesis Doctorales y comunicaciones de ICOM).

El objetivo final se focaliza en aportar una visión panorámica del estado de la cuestión de los principales estudios y tendencias que ayude a entender mejor la evolución del papel de la evaluación en esta línea de investigación asociada al campo de conocimiento de Didáctica de las Ciencias Sociales.

Se ha considerado oportuno acotar cronológicamente el período de publicaciones vinculantes al objeto de estudio a las últimas dos décadas, como la época de crecimiento y asentamiento de la EP. Este estudio quiere aportar una visión panorámica de la evolución del papel de la evaluación en este campo de conocimiento. Con un carácter eminentemente exploratorio y descriptivo que puede orientar los posibles cambios o necesidades asociadas a las iniciativas que se realizan en el campo de la EP.

Se realizó una revisión sistemática de documentos públicos en la web de ICOM y publicaciones de sus conferencias generales, como organismo internacional representativo del ámbito de museos, y aquellas procedentes de ICOM-CECA, Comité encargado del ámbito educativo y cultural en estas instituciones. Esta revisión se complementa con el análisis de los trabajos de investigación doctoral en la base de datos de TESEO para ofrecer una panorámica de la producción científica nacional. Para ello, se realiza un tipo de muestreo intencional que responde a criterios de selección para la revisión de abstracts y documentos completos con el fin de decidir si la información que contenían estaba o no relacionada con nuestro objetivo. Tras la búsqueda inicial se localizaron 245 estudios potenciales, se analizaron 36 ponencias, aunque sólo desarrollamos en este estudio 18 y 22 Tesis doctorales.

El estudio se ha planteado en varias fases: 
1) Revisión de las contribuciones de las conferencias y ponencias específicas de ICOM-CECA, con el fin de aproximarnos al panorama internacional de los estudios evaluativos en el campo de la EP (museos prioritariamente).

- Estrategia de búsqueda en la web institucional completada con la revisión de aquellas conferencias aún no publicadas en línea, pero disponibles en archivo propio de los autores. - En cuanto a los criterios de inclusión en la búsqueda se decidió a delimitar aquellas conferencias de ICOM, ICOM-CECA que hacían referencia explícita en el título a la evaluación o términos afines. Mismo criterio para las ponencias revisadas en archivo propio para efectuar el análisis de contenido tabulado en la figura 1.
2) Focalizar el estudio en la producción de tesis doctorales registradas en TESEO, de ámbito nacional, evidenciando aquellas que desarrollan el binomio EP y evaluación.

- La estrategia de búsqueda se desarrolló en la opción avanzada de la base de datos, en el menú buscar tesis con todas las palabras (términos específicos, que se detallan en el análisis) junto con la combinación de palabras claves, como: museología, pedagogía y ambos. No se han empleado ecuaciones de búsqueda compleja ni con operadores booleanos para refinar la recuperación de registros (Cordón, Vaquero Pulido y López, 2001). Véase diagrama de flujo del proceso de búsqueda. (figura 2).

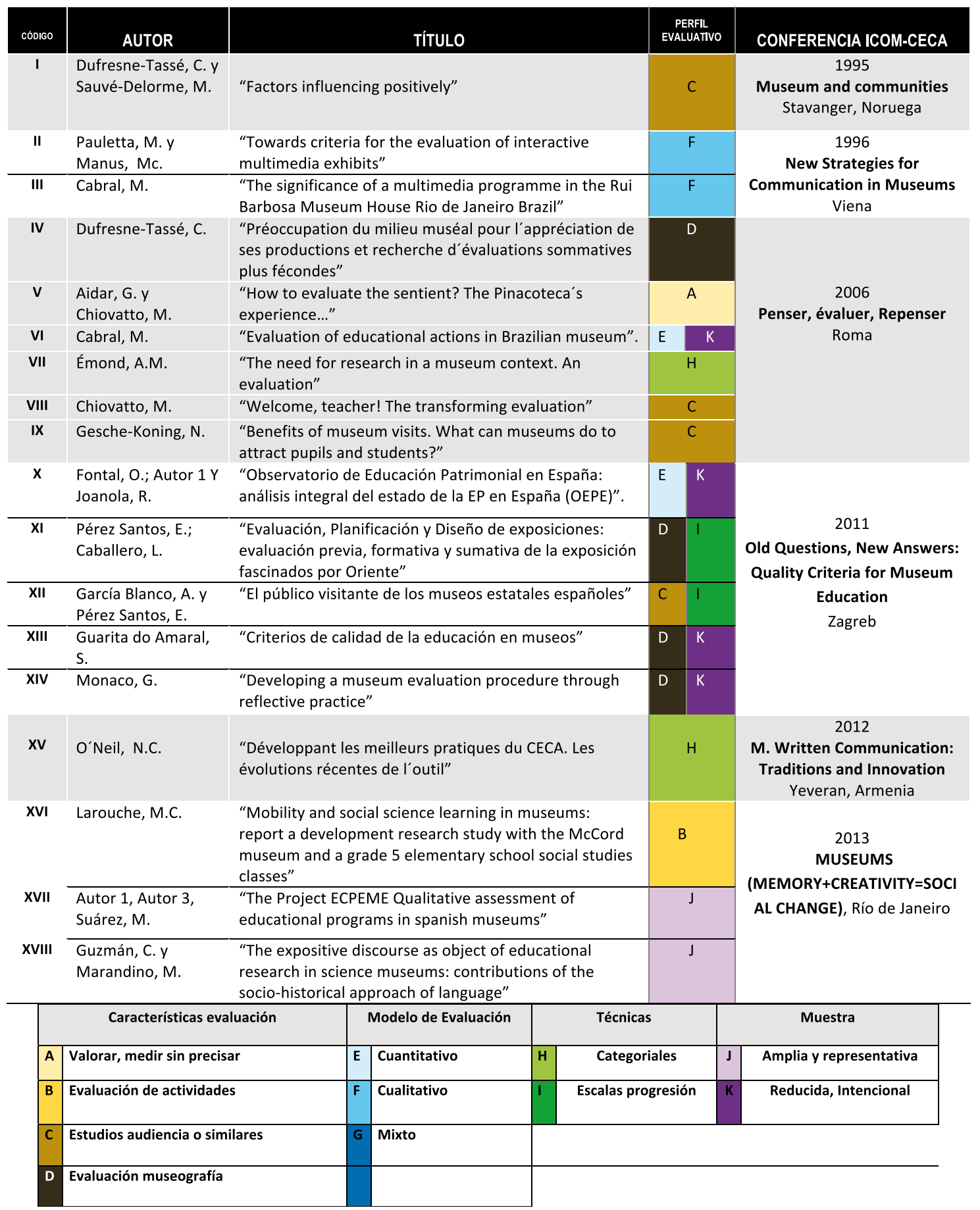

Figura 1. Perfil evaluativo conferencias y ponencias ICOM-CECA 
- Los criterios de inclusión fueron principalmente los conjuntos de descriptores: evaluación y patrimonio, y evaluación y museos, bien en el campo título, o en título y resumen. Como la recuperación de información produjo silencio, que, conociendo la producción científica en EP, no era verosímil. Se decidió a realizar un análisis de contenido de las tesis registradas en la búsqueda, incorporando el conocimiento informado de Roser Calaf como miembro de varios tribunales en la última década disponía del documento original que no se encuentra accesible en línea. Manteniendo el mismo criterio de inclusión. Como resultado se obtiene la rúbrica de evaluación de las Tesis Doctorales (figura 3).

La información analizada se ha estructurado en dos subapartados, uno dedicado a cada fase de análisis. Del conjunto de estudios analizados se extrajo la información de diferentes variables: autoría/organización, país, año, título, ámbito de estudio, finalidad evaluativa, modelo, metodología, técnicas y muestra.

\subsection{Análisis primera fase: Conferencias y ponencias ICOM}

Como se ha indicado el criterio de selección fue el título asignado a las Conferencias de ICOM. Siguiendo el mismo criterio para la revisión de las ponencias de cada anualidad publicada bien en web o monográfico. El término evaluación sólo se hace explícito en 2006, y conceptos afines como los empleados en ICOM-CECA 2011 (calidad), ICOM-CECA 2012 (innovación), incluso en ICOM-CECA 2013 (cambio social) como proceso que puede ser mediatizado por mecanismos de evaluación.

El perfil evaluativo de los estudios presentados en las ponencias ha venido designado por la tradición en evaluación educativa (De Miguel, 2000) que señalan como principales rasgos distintivos: finalidad, modelo, técnicas y muestra. Para ofrecer esa primera fotografía evaluativa de ICOM-CECA es representativa la figura 1.

La codificación y revisión documental parte de un primer nivel de identificación del tipo de evaluación desarrollado. En la asignación (A), se encuentran los estudios que no explicitan diseño evaluativo, ni técnicas de recogida de información. En el (B) vendría designado por aquellos estudios que plantean la evaluación enfocada a la actividad. Por su parte el (C) son evaluaciones que se ajustan a los modelos empleados en estudios de audiencias. Para completar el apartado, el código (D) agrupa los estudios distinguidos por las fases tradicionales en la evaluación de museos (previa, formativa, sumativa). (Calaf, 2017).

En segundo lugar, interesa conocer el enfoque metodológico de evaluación desarrollado. Para ello, (E) indica el carácter cuantitativo, (F) el carácter cualitativo o (G) mixto, de combinación metodológica. En el nivel $(\mathrm{H})$ interesa evidenciar el diseño evaluativo con uso de herramientas para catalogación y clasificación. Sin embargo, en el (I) la evaluación emplea estrategias más complejas como escalas progresivas, como las sugeridas por (Gutiérrez, 2019). A su vez, clasificamos los estudios en función de la muestra utilizada (J, K).

Es una evidencia la escasa presencia de la evaluación, cuando en el perfil domina la evaluación de estudios de audiencia y de exposiciones (códigos VIII y XVI). Existen evaluaciones complejas efectuadas desde grupos de investigación arraigados como GISEM, centradas en analizar procesos de enseñanza-aprendizaje por medio de modelos cualitativos (códigos VIII y XV). También existen diseños cuantitativos para valorar las actuaciones de escolares y su aprendizaje, manifestadas en las ponencias con vínculos con el Smithsonian Center (código XVII). Pero el mode- lo cualitativo es destacable en las intervenciones de la Universidad de Sao Paulo en colaboración con Museos de Brasil (Cabral, códigos III y X), así como en Gúzman y Mandarino (Código XVIII), o en Dufresne-Tasssé (Código IV).

\subsection{Análisis segunda fase: Tesis Doctorales de Educación Patrimonial en España}

Pero no debemos obviar que la mirada panorámica del avance científico de un área viene determinada por el desarrollo de investigación, y notablemente por las Tesis doctorales que ampara. En nuestro país debemos recurrir a la base de datos de TE$\mathrm{SEO}$, pero como cualquier sistema de recuperación de información, tiene sus limitaciones. En primer lugar, esta base de datos se ve condicionada por los descriptores de búsqueda que emplea el tesauro de la UNESCO. Diccionario específico de términos científicos tradicionales que no contempla el descriptor EP en el campo de búsqueda: palabra clave.

Un primer sondeo muestra ya la disparidad de términos empleados por los investigadores para asignar descriptores al reseñar sus tesis, lo que complica aún más una búsqueda refinada que muestre un fiel reflejo de la investigación desarrollada en este ámbito, lo que podría conllevar alguna omisión.

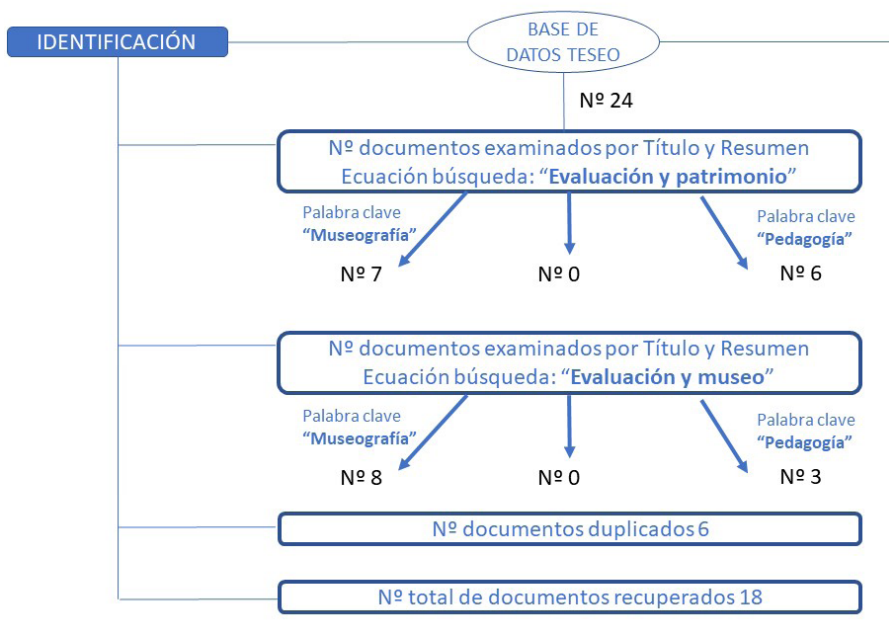

Figura 2. Diagrama de flujo del proceso de búsqueda avanzada en Teseo

La estrategia de búsqueda viene determinada por el uso de los diferentes comandos y campos de búsqueda para precisar la recuperación de la información relevante. En este sentido cuando no se introduce descriptor en el campo: palabra clave, el primer resultado que obtenemos apenas alcanza la decena de tesis y en la gran mayoría de ellas en su título aparece el término Educación Patrimonial. Los registros aumentan cuando utilizamos términos como Pedagogía o Museología, disciplinas con arraigo científico y nomenclatura UNESCO. Por otro lado, si la búsqueda se realiza sólo en el campo: título, el número de registros disminuye comparativamente a cuando se hace de forma simultánea como título y resumen. Los descriptores que recuperan un mayor número de registros son, por este orden: Patrimonio, educación y museos, Educación patrimonial y Didáctica patrimonio, cuando empleamos Museología como clave para filtrar resultados. Si utilizamos el término Pedagogía aparece algún registro sobre programa educativo, pero los descriptores más comunes son: Patrimonio, educación y museos, y Didáctica patrimonio.

La búsqueda simultánea en título y resumen es más resolutiva cuando se indica el término evaluación vinculada a las palabras clave: Museología y Pedagogía. 


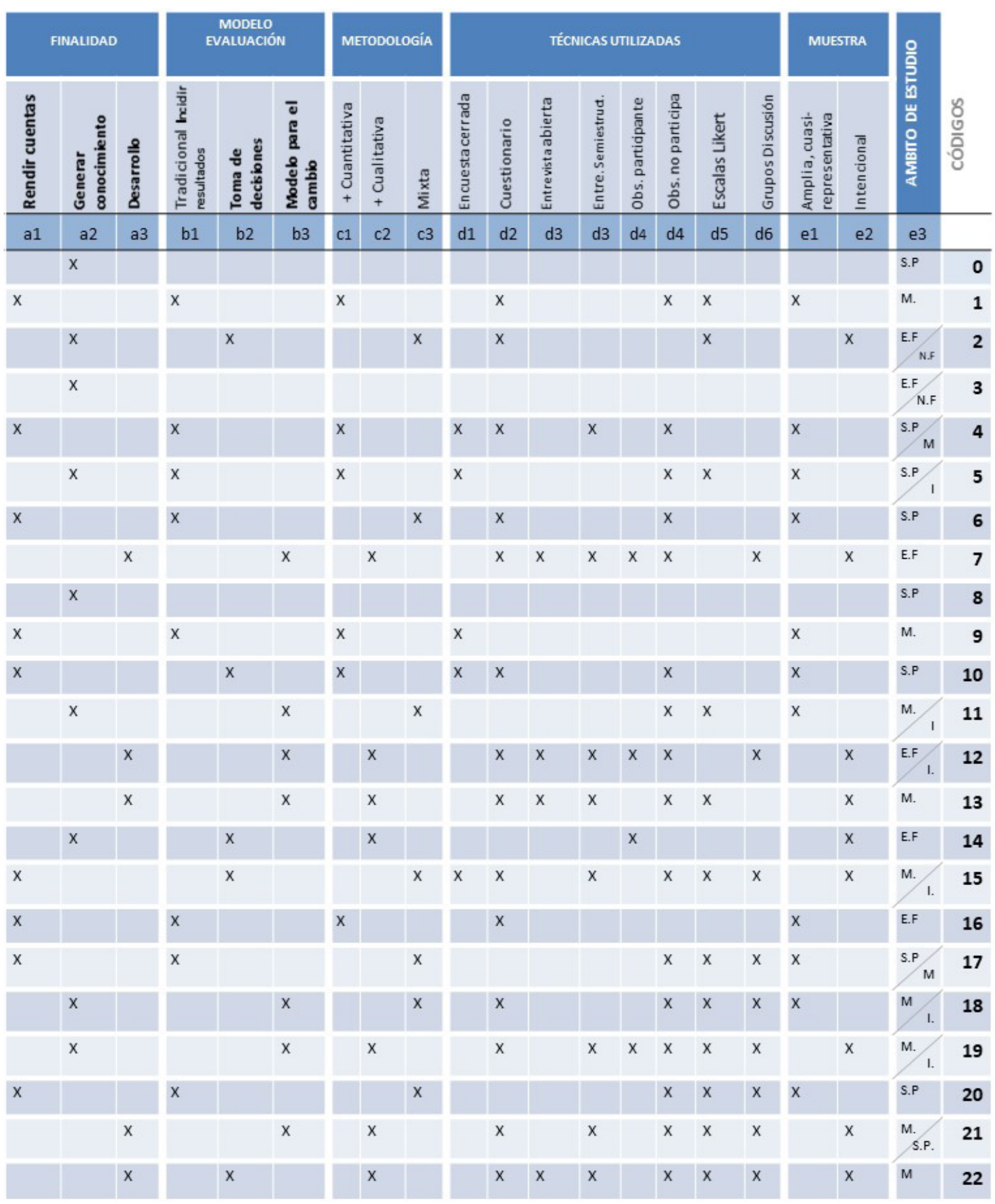

AMBITO:

E.F. Educación Formal

S.P. Sitios de Patrimonio I. Internety Giro tecnológico

N.F. Educación No Formal

M. Museos

Figura 3. Tesis y rúbrica de evaluación

La recuperación de registros por mecanismos lógicos de la base datos de TESEO vemos que no es eficaz a nuestro propósito siendo conocedores de la producción en el ámbito de la EP, existe silencio documental donde no debería de existir. Esta casuística ya orienta nuestra elección y procedimiento de análisis documental mediante el desarrollo genealógico. Nuestro análisis documental no sólo abarca los registros de tesis vinculadas a la evaluación de la EP sino también al contenido y temática de las mismas que permite definirlas, clasificarlas. Se opta por una interpretación genealógica a modo de mapa conceptual donde se perciben las aproximaciones a modelos evaluativos consensuados por la tradición en investigación evaluativa (figura 3) que facilita el análisis sobre: la finalidad, el modelo evaluativo utilizado, la muestra y las técnicas de recogida de la información.

En el eje vertical encontramos las tesis (enunciadas por el código reflejado en el anexo). Cada hilera de este cuadro nos ofrece una "radiografía sencilla" que se corresponde con una tesis analizada bajo la perspectiva de una interpretación evaluativa. 


\section{Resultados}

Si focalizamos el estudio en las publicaciones del Comité de Educación y Acción Cultural de ICOM en sus cuatro décadas de historia se ha preocupado por ofrecer un foro de encuentro y de intercambio de experiencias entre los profesionales de los museos y expertos investigadores del área. Por ello, el análisis de sus conferencias ha evidenciado la influencia de este organismo para avanzar líneas de investigación derivadas de las necesidades prácticas del museo.

La evaluación ha ido tomando fuerza, primero promoviendo la motivación de los visitantes y la exposición como medio de enseñanza (ICOM, 1995, 1996). Para explorar cómo desarrollar una evaluación de una visita al museo. Principalmente a través de revisiones bibliográficas y críticas de la evaluación en el museo para alcanzar criterios de mejora, que avanzan el tema de la conferencia de 2011: Criterios de calidad para la educación, con el fin de dar respuesta a la función socioeducativa del museo, a la par que mantener un óptimo nivel de desarrollo profesional. La principal contribución de ICOM-CECA en esta conferencia es el desarrollo de un modelo de Buenas Prácticas desarrollo por O`Neill y Dufresne-Tassé (2012). Propuesta que tiene una doble intención, incentivar la participación de los museos desde esta sección creada ad hoc, y aprovechar el foro de la conferencia para discutir y desvelar los criterios de mejora implementados en esos programas reconocidos como Best Practice.

Se pretende fomentar la evaluación como mecanismo de orientación de los procesos educativos en el museo desde un planteamiento compartido. De ahí el siguiente paso: delimitar factores para la comunicación eficaz, Conferencia de 2014. Para facilitar el compromiso de implementar una cultura evaluativa en los museos y programas educativos como estrategia de renovación su acción cultural.

En definitiva, las Actas de las Conferencias de ICOM-CECA recogen estudios que pretenden mejorar e innovar la práctica, a excepción de aquellos conferenciantes, algunos autores ya mencionados, procedentes de países con tradición evaluativa (Canadá, Estados Unidos, Reino Unido) donde la colaboración entre universidad y departamentos educativos es efectiva.

En cuanto a los resultados sobre las Tesis doctorales de Educación Patrimonial, el análisis genealógico evidencia un proceso lógico de construcción con las Tesis que hemos definido como pioneras. Una vez asentadas las bases conceptuales y la definición del campo de estudio aparecen los primeros modelos evaluativos en clave educativa que se diversifican en dos grupos. Por un lado, aquellos que buscan comprender el patrimonio en su contexto amplio, y otros que persiguen la mejora del aprendizaje escolar. Pero, ambos grupos responden al modelo tradicional de evaluación por objetivos, aunque algunos incentivan la toma de decisiones.

Una segunda fase se caracteriza por la significatividad que empieza a adquirir la evaluación en el ámbito de la EP, enfocada hacia la satisfacción de las audiencias o en la demostración de la eficacia de las intervenciones educativas. La finalidad es compartida, comprender el contexto y tomar decisiones que incentiven el cambio. En este período la diversidad metodológica se generaliza. Para finalmente, encontramos con un aparente arraigo evaluativo generado desde proyectos de investigación, desarrollo e innovación donde se enmarcan las tesis revisadas. Encontramos que se han adoptado dos modelos diferenciados: evaluación de estándares (OEPE), y evaluación cualitativa (ECPEME, EPITEC), planteamientos comple- mentarios enfocados al cambio y mejora de los programas de Educación Patrimonial, museos y sitios de patrimonio.

Por su parte el análisis de la rúbrica evaluativa permite profundizar en el perfil de las Tesis doctorales en EP. Cabe señalar que la categorización del perfil evaluativo es similar, con ligeras diferencias, a la empleada en las Conferencias ICOM-CECA. Al tratarse de documentación científica el nivel de codificación se ha incrementado. Siguiendo los criterios propios de la tradición en evaluación educativa (CITA), donde el nivel (A) responde a la finalidad evaluativa, distribuida en torno a la clasificación (rendición de cuentas, generar conocimiento y de desarrollo); en el nivel (B) se distinguen los modelos (tradicional, toma de decisiones o para el cambio), en el (C) metodología (cuantitativa, cualitativa o mixta), en el (D) especificación de las técnicas de recogida de información y (E) el tipo de muestra: intencional o representativa estadísticamente.

La panorámica global sobre los modelos de evaluación se encuentra distribuida en la tradicional rendición de cuentas, enfocada a objetivos, junto al interés de generar el campo teórico de referencia de la E.P (ejemplos: tesis de Cuenca (código 2) y Fontal (código 3). Siendo más escasas aquellas investigaciones que tienden al desarrollo educativo (ejemplos: código 7, 12, 13, 21 y 22). Como tesis de Myriam Martín (código13), desde su planteamiento metodológico consigue una proyección que marca una nueva tendencia hacia el cambio del fenómeno educativo en museos. Perspectiva, que en los últimos años va cogiendo impulso y continua $(18,19,21)$. Si el contexto objeto de estudio es la escuela, la evaluación adquiere un sentido similar, de cambio y carácter cualitativo (Monfort, código 7).

También se manifiesta una brecha tecnológica en esta producción, considerando el análisis y desarrollo de interactivos (TIC, apps,...) que sirven de apoyo educativo-expositivo, recursos sofisticados para mejorar la experiencia, como en lad tesis de Vicent (Código 13) o López Benito (código 18).

Al triangular la información de la rúbrica evaluativa (Figura 3) y el de interpretación genealógica (Figura 4) se manifiesta la filiación de los diferentes investigadores pioneros en el ámbito de la EP y su vinculación universitaria, y cómo han ido creando escuelas. La pluralidad académica desde la que se ha estudiado la Educación Patrimonial es causa de la diversidad de perfiles de directores y doctorandos: arqueólogos, licenciados en Bellas Artes, historiadores, geógrafos, pedagogos, psicólogos. En este sentido, la evaluación en educación patrimonial/didáctica del patrimonio no ha sido una necesidad en los años analizados, a excepción de aquellas Tesis donde el modelo de estudio de audiencias es el eje vertebrador de la investigación. Y también esta realidad poliédrica ha marcado una idiosincrasia distintiva de la Educación Patrimonial en España, donde la evaluación ha ido tomando impulso, ampliando marcos de acción-reflexión, donde los contextos educativos se disipan en pro del uso del patrimonio como mediador de la mejora en la acción educativa.

Tenemos las tesis que consideran factores relativos al proceso, visión comprensiva de todos los factores que intervienen en la calidad y consideran valoraciones sociales de la audiencia. Usan metodología mixta, con predominancia en la Universidad de Barcelona (grupo DIDPATRI). La visión integral de la evaluación donde el diseño y técnicas de evaluación cualitativas se materializa en el análisis del programa educativo del museo desde una perspectiva institucional de comunidad de aprendizaje escuela-museo (Calaf, Suárez y Gutiérrez, 2015). Perspectiva iniciada con la tesis de José María Cuenca (2002) que se ve ampliada con el modelo evaluativo ECPEME. 


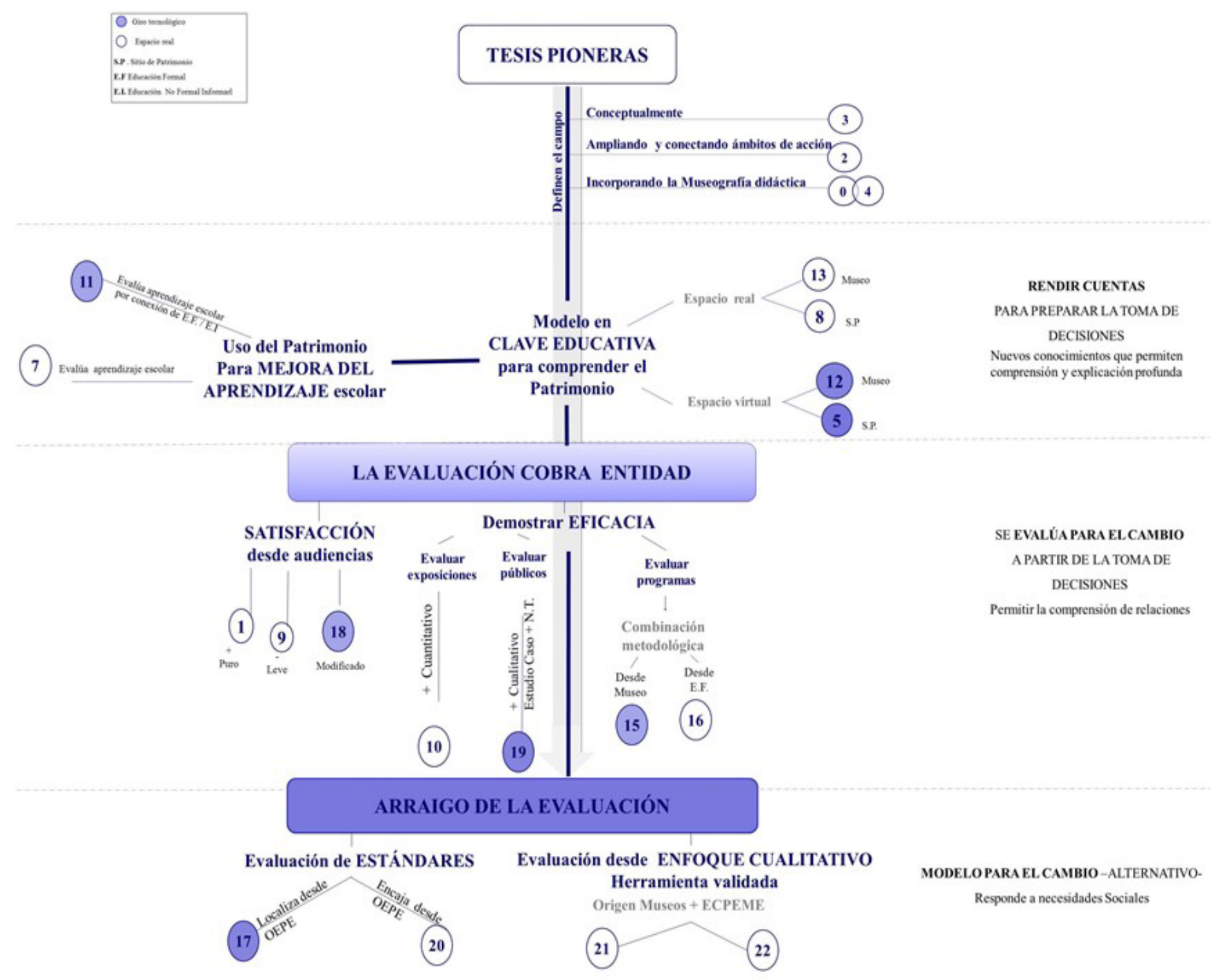

Figura 4. Mapa cognitivo sobre la genealogía de la relación EP-Evaluación

\section{Discusión}

Los primeros resultados obtenidos sobre las Conferencias y Congresos ICOM, podrían definirse cómo la evaluación trata de naturalizarse con el apoyo de organismos internacionales. Parece manifestarse que no todo es evaluado en el ámbito educativo de los museos y sitios de patrimonio en el contexto global.

En la década de 1970, desde Estados Unidos, se optimizan esfuerzos para ofrecer un corpus técnico-sistemático, donde la evaluación se institucionaliza dos décadas más tarde y en Europa se crean los Observatorios de Públicos. En el contexto europeo la creación del Consejo Internacional de Museos (ICOM) será el organismo encargado de protagonizar la salvaguardia y difusión del patrimonio, tras finalizar la Segunda Guerra Mundial (1946-1947). En sus inicios la preocupación recaía, dada la época, en la conservación, restauración y libre circulación de los bienes culturales (ICOM, 1950). Las siguientes dos décadas responden a un propósito profesionalizante. Se concatenan propuestas acerca del rol educativo y cultural del museo, así como las conferencias de los años 80 vendrán marcados por la res- ponsabilidad y compromiso social del museo como promotores de intercambio y desarrollo cultural. Y donde queda reflejado el componente educativo en la evaluación en el museo desde las primeras intervenciones de Screven en la Conferencia de 1985. Es a partir del nuevo milenio, donde el Comité apuesta por convertir a los museos en un agente de cambio (ICOM, 2001, 2010 , 2013). Esta última Conferencia vendría abanderada por la accesibilidad e exclusividad del museo no sólo desde aspectos arquitectónicos sino también de compromiso educativo de un Diseño para todos y donde la evaluación va tener una finalidad instrumental.

Existen evidencias de la relación entre EP y evaluación, donde se constata que hasta fechas recientes el interés científico no recaía en este ámbito, tal y como se ha puesto de manifiesto tanto en la documentación de ICOM-CECA como en la muestra de tesis doctorales de los últimos 20 años. No obstante, es cierto que destaca una evolución metodológica y de modelos evaluativos, que algunos autores reclamaban (Shettel, 1990, 2001; Pérez Santos, 2000, Fontal e Ibáñez-Etxeberría, 2017). En el caso de los museos, la evaluación de audiencias ha sido la dominante, seguida 
de la evaluación de los aprendizajes adquiridos, en el caso de los programas. Poco a poco se ha ido incorporando perspectivas más comprensivas orientadas a la evaluación de los programas -en su diseño, procesos y resultados- y se han ampliado los contextos institucionales objeto de estudio en que se desarrollan. Existe una representatividad significativa de la diversidad paradigmática y metodológica adoptada (cualitativas y cuantitativas, descriptivas y experimentales...). En España, el modelo ECPEME ejemplifica este tránsito de los modelos de evaluación de públicos y/o de adquisición de aprendizajes a un modelo de evaluación de programas en un contexto de colaboración interinstitucional empleando una complementariedad metodológica (Gutiérrez, 2019). La evaluación de programas no ha tenido el mismo arraigo que la evaluación de exposiciones (Bitgood y Shettel, 1996; Asensio, Pol y Gomis, 2001) aunque la tendencia está cambiando en los últimos años (Fontal e Ibáñez-Etxeberría, 2017).

Comprobar el binomio de evaluación y educación patrimonial en museos ha evidenciado la función más instrumental de la evaluación relacionada tradicionalmente con las tareas de catalogación e inventariado (McManus, 2016). Sin embargo, a medida que la función educativa se ha arraigado en los museos los planteamientos evaluativos han transitado por dos fases principalmente. Primero, adoptando parámetros propios de la estructura curricular (Monfort, código 7), para continuar ensayando y elaborando modelos propios ad hoc adaptados a contextos diversos de Educación Patrimonial. (Calaf y Suárez, 2016).

La aproximación de la educación formal al patrimonio y viceversa ha contribuido a extender las prácticas evaluadoras en dicho ámbito, esta alianza no siempre ha actuado como factor de innovación. Paradójicamente, va a ser en la educación no formal donde surgen con más frecuencia innovaciones en el ámbito de la enseñanza y la evaluación del patrimonio (Asensio, 2015). Durante los últimos años, la preocupación por la calidad, en sus diversas acepciones, ha llevado también a un incremento de las iniciativas de evaluación en el ámbito de los museos (O’Neill \& Dufresne-Tassé, 2011). Y “es en este contexto que debemos leer la persistencia de las metodologías de enfoque público" y averiguar en qué medida están destinadas a la democratización cultural o si pretenden el renacimiento de anteriores paradigmas (Romanello, 2012: 19). Frente a los modelos productivos -centrados sobre la cuantificación de la asistencia, índices de satisfacción, rendimiento de cuentas- (Pérez Santos,2000) y reproductivos -adquisición de contenidos y conceptos patrimoniales-,están surgiendo otras tendencias más preocupadas por la igualdad, la transformación social y la dimensión afectiva e inclusiva que la EP puede aportar al aprendizaje y desarrollo integral de las personas, en línea con las nuevas corrientes de la investigación evaluativa presentes en la conferencia ICOM-CEA, Washington, 2015 (ej: Chiovatto; Meunier y Dusfresne Tasé, Cabral).

\section{Conclusiones}

Como consecuencia de la relación escuela-museo, hay un período en el que la escuela se llegó a beneficiar de esta vinculación en términos didácticos. Sin embargo, el museo limitó el enfoque educativo de su evaluación al reproducir el modelo tradicional escolar dirigido a comprobar la adquisición de conocimientos sobre el patrimonio (Nardi, 2007). Si bien es cierto que la evaluación puede gozar de esa potestad, su "poder" debe ir más allá de comprobar objetivos y contenidos, persiguiendo el desarrollo de conocimiento y mejorar la realidad - procesos, relaciones- en la que se circunscribe (De Miguel, 2000; Santos
Guerra, 2003, entre otros). Entender la evaluación como un proceso más de la intervención educativa, como un continuum, y no como la fase final de la acción didáctica. Incluso concebirla como un proceso más de investigación. El desarrollo histórico de la evaluación educativa determina su integración en el marco organizativo institucional en el que se desarrolla dicha acción educativa. Evaluar programas y servicios sociales es una política necesaria que contribuye a dar sentido a los propios programas (San Fabián, 2014: 43).

Pero se impone la realidad económica de los últimos años, las demandas sociales y educativas, que provocan colaboraciones necesarias y esperadas como las relatadas por Barbara Franco (2010), donde maestros y educadores de museo se enfrentan $a$ tratar de involucrar a los estudiantes en experiencias activas, al mismo tiempo que cumplen los requisitos de estándares de desempeño (p.230). Surgen fenómenos que no entienden de fronteras epistemológicas de la ciencia, como la estandarización, que afectan a ambos contextos (museos y escuelas). A nivel institucional los museos y sitios de patrimonio se encuentran subyugados por la asignación de fondos, en muchos casos, según el tamaño de la audiencia; por tanto, los estudios de visitantes se convierten en la herramienta perfecta para demostrar su "éxito" y de obligado cumplimiento para una adecuada gestión (McManus, 2016: xiii). Los estudios de público son una herramienta gerencial perfecta (Alarcón, 2005) o de gestión de las exposiciones, en particular, para su función comunicativa (Pérez Santos, 2000: 17) ¿Y para su función educativa? La exposición, sus objetos, transmiten información con su mera presencia, pero el acto educativo precisa de códigos y estrategias que eleven esa información a conocimiento (Calaf, 2017).

Como se ha mostrado la Educación Patrimonial está abierta al cambio respondiendo de manera paulatina a las nuevas demandas socioeducativas y tecnológicas. Si el deseo es continuar este progreso debemos someter nuestros avances a procesos sistemáticos de revisión y evaluación. Para ello, algunas de las líneas de futuro debieran considerar:

- Incorporar nuevos diseños y técnicas para el desarrollo de evaluaciones de carácter integral (auto-informes, etnografía digital).

- Proporcionar otro tipo de evaluaciones más participativas, de implicación de todos los agentes educativos, orientadas a descubrir el contexto, proceso y resultados de aprender y vivir el patrimonio (iluminativa), a dar respuesta a las necesidades del contexto (sensible a la diversidad) y a transformar las condiciones de vida objetivas y subjetivas de la comunidad de aprendizaje -museo-, desde un planteamiento más crítico. Integrando la evaluación en el diseño de los programas.

- La colaboración entre Universidades y profesionales podría favorecer mecanismos de evaluación que permitan establecer un equilibrio entre mejora interna y funcionalidad pública (autoevaluación institucional, evaluación externa).

- Aplicar sistemas de metaevaluación que permitan seguir aprendiendo de los procesos evaluadores.

El papel que tiene la evaluación en el contexto actual es responder de manera realista a las necesidades para mediar en el cambio socioeducativo del contexto real en el que se desarrolla la intervención con propuestas coherentes y ajustadas a la realidad (De Miguel, 2015). Por tanto, el nuevo binomio a experimentar es la investigación y la evaluación como funciones complementarias para el avance de la Educación Patrimonial. 


\section{Referencias bibliográficas}

Alarcón, R. (2005). Una lectura desde los estudios de públicos. Museo: Revista de la Asociación Profesional de Museólogos de España, 13, 290-295. Obtenido de: https://docplayer. es/7664855-Una-lectura-desde-los-estudios-de-publicos.html

Alarcón, R. (2007). Sociología y estudios de público en los museos españoles. En Museo: Revista de la Asociación Profesional de Museólogos de España, 12, 233-246. Obtenido de: http:/ / bit.ly /2nZM2nj

Angulo, N. (2009). ¿Qué son los observatorios y cuáles son sus funciones?. En Innovación Educativa, 9 (7), 5-17. Obtenido de: http:/ / bit.ly/2oyo8zE

Asensio, M., y Martín, E. (2015). Evaluación (implicativa) de programas (colaborativos) en el museo (participativo): una reflexión de caso sobre el proyecto Conect@ (pp. 175-197). En S. González (Coord.) Un proyecto de colaboración entre personas con diferentes capacidades para promover la plena accesibilidad a la cultura. Obtenido de: http:/ / bit.ly/2Vfgitx

Asensio, M., Pol, E. y Gomis, M. (2001). Estudios de público, evaluación de exposiciones y programas y diseño de áreas expositivas en el Museu Marítim. Drassana: revista del Museu Marítim, 9, 18-31. Obtenido de: http://bit.ly/2XAyHil

Bitgood, S. (2011). Social Design in Museums: The Psychology of Visitor Studies: Collected Essays. Volume One. Edimburgo: Museums.[re-edición]

Bitgood, S. y Shettel, H. (1996). Determining Museum Effectiveness: Visitor Studies Today. The Journal of Museum Education, 3 (21), 6-10 Obtenido de: https://www.jstor.org/stable/40479068

Calaf, R. y Suárez, M. A. (Coords.). (2016). Acción educativa en museos su calidad desde la evaluación cualitativa. Gijón: Trea

Calaf, R., Suárez, M. A y Gutiérrez, S. (2015). La evaluación de la acción cultural en museos. Gijón: Trea

Cordón, J. A., Vaquero Pulido, J. R., y López, J. (2001). Manual de Investigación Bibliográfica y documental. Teoría y Práctica. Madrid: Pirámide

Cuenca, J.M. (2002). El patrimonio en la didáctica de las ciencias sociales. Análisis de concepciones, dificultades y obstáculos para su integración en la enseñanza obligatoria. (Tesis doctoral inédita). Universidad de Huelva.

Cuenca, J.M., Estepa, J. y Martín, M. (2013). ¿Qué visión del patrimonio se transmite en los museos andaluces? Imagen e identidad de Andalucía (pp. 105-128). En J. Estepa (Coord.). La educación patrimonial en la escuela y el museo: investigación y experiencias, $105-128$.

Daignault, L. (2011). (Ed.). L'évaluation muséale : savoirs et savoirfaire. Québec: Presses de l'Université du Québec.

De Miguel, M. (2000). La evaluación de programas sociales: Fundamentos y enfoques teóricos. Revista de Investigación Educativa,18 (2), 289-317.

De Miguel, M. (2015). Ideología y pedagogía empírica: cuestiones para un debate. Revista de Investigación Educativa, 33(2), 269-287.

Fontal, O. (2003). La educación patrimonial: definición de un modelo integral y diseño de sensibilización. Tesis Inédita. Universidad de Oviedo.

Fontal, O. (2014). La educación patrimonial en España necesidades e ilusiones para la próxima década. $\mathrm{PH}$ : Boletín del Instituto Andaluz del Patrimonio Histórico, 22, 85, 12-14. Obtenido de: http:/ /bit.ly/2nRYDJx

Fontal, O. (2016). The Spanish Heritage Education Observatory/ El Observatorio de Educación Patrimonial en España, Cul- tura y Educación. En Cultura y Educación, 28, 254-266, DOI: http:/ /dx.doi.org/10.1080/11356405.2015.1110374

Fontal, O. y Marín, S. (2011). Enfoques y modelos de educación patrimonial en programas significativos de OEPE. Educación artística y revista de investigación, 2, 91-96, DOI: https://doi. org/10.7203/eari.2.2508

Fontal, O. e Ibáñez, A. (2015). Estrategias e instrumentos para la educación patrimonial en España. Educatio Siglo XXI, 33, (1), 15-32, DOI: https://10.4438/1988-592X-RE-2016-375-340.

Fontal, O. e Ibáñez-Etxeberría, A. (2017). La investigación en Educación Patrimonial. Evolución y estado actual a través del análisis de indicadores de alto impacto. En Revista de Educación, 375, 184-214. Obtenido de: https://sede.educacion. gob.es/publiventa/descarga.action?f codigo agc $=18213$

Fontal, O.; Rodríguez, M. y Macías, I. (2017). Trayectoria y prospectiva estratégica del Observatorio de Educación Patrimonial en España. En Fontal, O.; Ibáñez-Etxeberria, A.; Domingo, M. y Marín, S. Comunicaciones del III Congreso Internacional de Educación Patrimonial, 62-71. Obtenido de: http://bit. ly/2oyAt6X

Franco, B. (2010). Advocacy for education in museums. In Journal of Museum Education, 35(3), 229-235. Obtenido de: https:// doi.org/10.1080/10598650.2010.11510670

Gutiérrez, S. (2019). Evaluación cualitativa de programas educativos en museos de patrimonio artístico (Asturias y Oporto). Tesis doctoral inédita. Universidad de Oviedo.

ICOM. (1950). Exchange of colletions and conservation personnel; inventory of scientific instruments; museums and education; problems of professional training. London

ICOM. (1971). The museum in the service of man, today and tomorrow: the museum's educational and cultural role. Paris.

ICOM. (1974). The museum and the modern world. Copenhagen.

ICOM. (2001). Managing Change: museums facing economic and social challenges. Barcelona.

ICOM. (2010). Museums for Social Harmony. Shanghai.

ICOM. (2013). Museums (memory + creativity) = social change. Rio de Janeiro.

ICOM-CECA. (1985). L'éducateur de musée et la recherche. Barcelone. Obtenido de: http://bit.ly/2oH3fT7

ICOM-CECA. (1997). Évaluation de l>éducation muséale et de l>action culturelle - Théorie et pratique. Rio de Janeiro. Obtenido de: http://bit.ly/2oH3fT7

ICOM-CECA. (2006). Penser, Évaluer, Repenser. Rome.

ICOM-CECA. (2011). Old Questions, New Answers: Quality Criteria for Museum Education. Zagreb. Obtenido de: http://bit. ly $/ 2 \mathrm{oH} 3 \mathrm{fT} 7$

ICOM-CECA. (2012). Museums and Written Communication: Traditions and Innovation.Yerevan. Obtenido de: http://bit. ly/2oH3fT7

ICOM-CECA. (2013). Museums (memory + creativity) = social change. Rio de Janeiro. Obtenido de: http://bit.ly/2oH3fT7

ICOM-CEA. (2015). Museum Education and Accessibility: Bridging the Gaps. Washington, D.C. Obtenido de: http://bit.ly/2oH3fT7

Llonch, N. y Santacana, J. (2011). Claves de la museografía didáctica. Lleida: Milenio.

Mark, M.M.; Henry, G. T.; Julnes, G. (2000). Evaluation: An Integrated Framework for Understanding, Guiding, and Improving Policies and Programs. Jossey-Bass: San Francisco.

McManus, P. (1996). Archaeological displays and the public: museology and interpretation. Institute of Archaeology, University College London: London.

McManus, P. (2016). Archaeological displays and the public: museology and interpretation. Routledge. 
Martín Cáceres, M. (2012). La Educación y la Comunicación Patrimonial. Una mirada desde el Museo de Huelva. Tesis doctoral inédita. Universidad de Huelva.

Nardi, E. (2007). Pensare, valutare, ripensare : la mediazione culturale nei musei. Milano : F. Angeli.

O’Neill, M.C. y Dufresne-Tassé, C. [2012]. Best practices in museum education and cultural programmes. Planning, developing and evaluating a programme. ICOM-CECA. Obtenido de: http:// bit.ly/2I4FmfB

Pérez Santos, E. (2000). Estudio de visitantes en museos: metodología y aplicaciones. Gijón: Trea.

Powell, L. H. (1938). A Study of Seasonal Attendance at a Mid-Western Museum of Science. En Museum News, 16(3), 7-8.

Romanello,G. (2012). Merci pour votre collaboration. Quelques réflexions sur les modalités d'approche des publics dans les institutions d'art contemporain. In Marges [En ligne], 15. Obtenido de: https://journals.openedition.org/marges/353

San Fabián, J.L. (2014). Evaluar programas socioeducativos en tiempos de crisis. Un enfoque organizacional. Oviedo: Trabe.

Santacana, J. y Serrat, N. (Coords.). (2005). Museografía Ddiáctica. Barcelona: Ariel

Santos Guerra, M.Á. (2003). Una flecha en la diana: la evaluación como aprendizaje. Madrid: Narcea.

Schoutenn, P. (1988). Psicología I disseny de les exposicions. En ICOM-CECA. La investigación del educador de museos. Conferencia ICOM-CECA. Serie de Investigación Museística, 2. Obtenido de: http:/ / bit.ly /2GEQkYt, 39-42.
Screven, C. (1976). Exhibit Evaluation. A Goal Referenced Approach. In Curator The Museum Journal, 19, (4), 271-290. Obtenido de: http://bit.ly/2nZNZ37

Screven, C. (1988). Exposicions educatives per a visitants sense guia. En ICOM-CECA. La investigación del educador de museos. Conferencia ICOM-CECA. Serie de Investigación Museística, 2. Obtenido de: http:/ / bit.ly/2GEQkYt, 43-64.

Screven, C. (1990). Uses of Evaluation Before, During and After Exhibit Design. In ILVS Review 1(2), 36-66. Obtenido de: http://bit.ly/2Ut47Vu

Scriven, M. (1967). The methodology of evaluation. En R. W. Tyler, R. M. Gagne y M. Scriven (eds.). Perspectives of curriculum evaluation, 39-83.

Shettel, H. (1973). Exhibits: Art form or educational medium? In Museum News, 52, (1), 32-41. Obtenido de: http://bit. ly/2UMxh72

Shettel, H. (2001). Do we know how to define exhibit effectiveness?. In Curator The Museum Journal, 44, 4,327-334. http:// bit.ly/2oFRaxu

Suárez, M.A. (2015). ¿Aprender el patrimonio o aprender con el patrimonio?. En M. A. Suárez, R. Calaf, y Gutiérrez, S. La evaluación de la acción cultural en museos. (pp. 35-49). Gijón: Trea

Suárez, M. A. (2017). Enseñar y aprender Historia con el patrimonio. Evaluación cualitativa en museos de Asturias. Tesis doctoral inédita. Universidad de Oviedo.

Anexo. Muestra de Tesis doctorales analizadas

\begin{tabular}{|c|c|c|c|c|c|}
\hline CódiGo & AÑo & TíTULO DE LA TESIS & INVESTIGADOR/A & Director/a TESIS & UNIVERSIAD \\
\hline 0 & 1994 & "Didáctica del patrimonio arqueológico: el proyecto del poblado ibérico de Alorda Park o les Toixoneres de Calafell" & Joan Santacana & Isidoro González & Valladolid \\
\hline 1 & 1998 & "Evaluación psicológica en los museos y exposiciones: fundamentación teórica y utilidad de los estudios de visitantes" & Eloísa Pérez Santos & $\begin{array}{l}\text { Carme Prats y } \\
\text { Manuel Muñoz }\end{array}$ & UM \\
\hline 2 & 2002 & $\begin{array}{l}\text { "El patrimonio en la didáctica de las ciencias sociales. Análisis de concepciones, dificultades y obstáculos para su } \\
\text { integración en la enseñanza obligatoria" }\end{array}$ & J. Mạ Cuenca & Jesús Estepa & Huelva \\
\hline 3 & 2003 & "La educación patrimonial: definición de un modelo integral y diseño de sensibilización" & Olaia Fontal & Roser Calaf & Oviedo \\
\hline 4 & 2005 & "Acciones didácticas en el marco de los museos. Estado de la cuestión y análisis" & Nuria Serrat & $\begin{array}{l}\text { Joan Santacana } \\
\text { Nuria Rajadell }\end{array}$ & Barcelona \\
\hline 5 & 2005 & "El factor didàctic en els centres d'interpretació virtual. El cas d'Atapuerca" & Rafael Sospedra i Roca & $\begin{array}{l}\text { Xavier Hernández } \\
\text { Cardona }\end{array}$ & UB \\
\hline 6 & 2006 & "Educación y patrimonio: El caso de los campos de trabajo de la comunidad autónoma del País Vasco" & Alex Ibañez Echebarria & J.M Correa Gorospe & UPV \\
\hline 7 & 2007 & "L'us didàctic i el valor educatiu del patrimoni cultural" & Neus Montfort & Joan Pagés & UAB \\
\hline 8 & 2007 & "Anàlisi dels espais de presentació arqueològics de l'Edat dels Metalls." & Clara Masriera & Joan Santacana & U.B \\
\hline 9 & 2010 & "Relación museo-territorio e implementación didáctica: Museo de la Escuela Rural de Asturias" & Marta García Eguren & Roser Calaf & Oviedo \\
\hline 10 & 2011 & $\begin{array}{l}\text { "Actividades educativas y didáctica del Patrimonio en las ciudades Españolas. Análisis, estado de la cuestión y } \\
\text { valoración para una propuesta de modelización" }\end{array}$ & Laia Coma Quintana & Joan Santacana & Barcelona \\
\hline 11 & 2011 & $\begin{array}{l}\text { "Educació patrimonial mitjançant recursos educatius en línea amb contingut de patrimoni cultural i xarxes d' } \\
\text { aprenentatge" }\end{array}$ & Janine Sprünker & Gloria Munilla & UOC \\
\hline 12 & 2012 & $\begin{array}{l}\text { "La cibermuseografía didáctica como contexto educativo para la E-A del patrimonio .Estudio de las páginas web } \\
\text { educativas de Museos virtuales de Arte" }\end{array}$ & Carmen Tejera & Rosa Mã Ávila & Oviedo \\
\hline 13 & 2012 & "La educación y la comunicación patrimonial: una mirada desde el Museo de Huelva" & Myriam Martin & José María Cuenca & Huelva \\
\hline 14 & 2013 & "Procesos de patrimonialización en el arte contemporáneo: diseño de un artefacto educativo para la identización" & $\begin{array}{l}\text { Carmen Gómez } \\
\text { Redondo }\end{array}$ & Olaia Fontal & Valladolid \\
\hline 15 & 2013 & "Evaluación de un programa de educación patrimonial basado en tecnología Móvil" & Naira Vicent & $\begin{array}{l}\text { MiKel Asensio } \\
\text { Alex Ibáñez }\end{array}$ & UAM \\
\hline 16 & 2014 & $\begin{array}{l}\text { "Programas de educación patrimonial en contextos informales: análisis y Valoración de su influencia en el alumnado } \\
\text { de eso de la zona minero- industrial de Bizkaia" }\end{array}$ & Iratxe Guillate Aierdi & $\begin{array}{l}\text { J. M. Madariaga } \\
\text { Alex Ibáñez }\end{array}$ & País Vasco \\
\hline 17 & 2014 & $\begin{array}{l}\text { "Educación patrimonial y diversidad: Evaluación de programas y definición de un modelo basado en los procesos de } \\
\text { Patrimonialización" }\end{array}$ & Sofía Martín Cepeda & Olaia Fontal & Valladolid \\
\hline 18 & 2014 & $\begin{array}{l}\text { "Aprendizaje Informal y Nuevas Tecnologías: Análisis y medición del constructo de interactividad en contextos de } \\
\text { exposición del patrimonio" }\end{array}$ & $\begin{array}{l}\text { Ma Elena Asenjo } \\
\text { Hernanz }\end{array}$ & Mikel Asensio & UAM \\
\hline 19 & 2014 & "Estudio exploratorio sobre la interpretación didáctica del arte en el museo a través de tecnologías móviles" & Victoria López Benito & Joan Santacana & UB \\
\hline 20 & 2016 & "Memoria, identidad y comunidad: evaluación de programas de educación patrimonial en la comunidad de Madrid" & Ana Sánchez Ferri & Olaia Fontal & Valladolid \\
\hline 21 & 2017 & "Enseñar y aprender Historia con el Patrimonio: Evaluación Cualitativa en Museos de Asturias" & $\begin{array}{l}\text { Miguel A. Suárez } \\
\text { Suárez }\end{array}$ & Roser Calaf & Oviedo \\
\hline 22 & 2019 & "Evaluación Cualitativa de Programas Educativos en museos de patrimonio artístico (Asturias y Oporto) & Sué Gutiérrez Berciano & Roser Calaf & Oviedo \\
\hline
\end{tabular}

